\title{
Estimating the permeability of cement pastes and mortars using image analysis and effective medium theory
}

\author{
H.S. Wong ${ }^{\mathrm{a}^{*}}$, R.W. Zimmerman ${ }^{\mathrm{b}}$ and N.R. Buenfeld ${ }^{\mathrm{a}}$ \\ ${ }^{a}$ Concrete Durability Group, Department of Civil and Environmental Engineering, Imperial College London \\ ${ }^{b}$ Department of Earth Science and Engineering, Imperial College London
}

\begin{abstract}
A method to estimate permeability of cement-based materials using pore areas and perimeters from SEM images is presented. The pore structure is idealised as a cubic lattice having pores of arbitrary size. The hydraulic conductance of each pore is calculated using the hydraulic radius approximation, and a stereological factor is applied to account for the random orientation of the image plane. A 'constriction factor' is applied to account for variations in pore radius along the pore axis. Kirkpatrick's effective medium equation is then used to obtain an effective pore conductance, from which the macroscopic permeability is derived. The method was tested on forty-six pastes and mortars with different $\mathrm{w} / \mathrm{c}$ ratio, cement, age and sand content. The permeabilities ranged from $3 \times 10^{-}$ ${ }^{18} \mathrm{~m}^{2}$ to $5.8 \times 10^{-16} \mathrm{~m}^{2}$. It was found that $76 \%$ of the permeabilities were predicted to within a factor of \pm 2 , and $98 \%$ within a factor of \pm 5 from measured values.
\end{abstract}

Keywords: Backscattered electron imaging (B); Image analysis (B); SEM (B); Porosity (B); Permeability, Effective medium theory

\section{Introduction}

This study concerns the use of images of the microstructure as a starting point to predict the rate of mass transport in cement-based materials, and ultimately, to predict the rate of deterioration and service life. The ability to estimate transport properties would be of great value, because laboratory measurements are expensive and timeconsuming. Conventional laboratory testing of permeability that involves applying a pressure gradient and measuring the fluid flux through the sample is a slow process, particularly for samples with low permeability, because of the long time required to condition samples and to obtain measureable flow at steady state. Several novel techniques such beam bending, thermopermeametry and dynamic pressurisation have been proposed for rapid permeability testing of saturated cement pastes, but these methods are generally not practical for mortars and concretes [1]. Their principles, relative merits and limitations are reviewed in Scherer et al. [1]. Since transport properties are essentially governed by the pore geometry, it follows that it should be possible to estimate transport properties from quantifiable attributes of the pore structure. Indeed, there are many examples of such

\footnotetext{
* Corresponding author: Tel: +44 (0)20 7594 5956; Fax: +44 (0)20 72252716

E-mail address: hong.wong@imperial.ac.uk
} 
work, particularly in geological sciences, where the focus is on understanding the transport of oil and gas in hydrocarbon reservoirs, and contaminants and solutes in rock formations.

An important issue is identifying the pores and size range that are most relevant to transport. The effect of capillaries, 'gel' pores, air voids, hollow-shell pores, cracks and their relative contribution to transport needs to be determined. Concretes contain micro-cracks originating from drying shrinkage, thermal actions or mechanical loading. It is expected that microcracks facilitate transport, and that when they form interconnected channels, their effect will be large. The aggregate-paste 'interface transition zone' (ITZ) is another feature of interest. Although the ITZ contains higher porosity than the 'bulk' paste region farther away from the aggregate, its net effect on transport is small, even when the ITZ fraction is sufficiently high to be considered overlapping [2]. An appropriate model would allow the effects of individual aspects of pore structure, that cannot be isolated in laboratory tests, to be investigated and quantified.

A range of modelling techniques is available to estimate transport properties from pore structure. The simplest approach is the classical Kozeny-Carman model [3, 4] that requires only two parameters that are averaged over the entire pore space: the porosity and specific surface. This model generally works well for unconsolidated and granular media, but loses accuracy for materials with a wide range of pore sizes, such as concrete. At the most fundamental level, transport coefficients can be derived from flow simulations through the actual 3D pore space. The governing equations are the Laplace equation for calculating diffusivity or conductivity, and the NavierStokes equations for calculating permeability.

Another approach is to convert the pore space into a 'resistor network' and then solve for the flow that results from an applied potential gradient (hydraulic, electrical, concentration) across the network. The conductivity of each pore channel can be estimated for irregular pores [5, 6] and if the network topology is known, the problem is reduced to solving Kirchhoff's equations for the network, i.e. a large, sparse system of linear equations. This can be done explicitly [7], but at the cost of extensive computations. In recent years, the second author of this paper, and his collaborators, have developed a method using Kirkpatrick's effective medium approximation to obtain accurate permeability estimates, which uses the same input data as do the explicit methods, but requires the solution of only a single nonlinear algebraic equation.

The method requires only the areas and perimeters of pores, as measured from images, as inputs, and is not computationally intensive. It is a refinement of previous models by Koplik et al. [8] and Schlueter [9], the principal difference being the application of stereological corrections to convert apparent pore geometries measured from a random 2D section to determine the true cross-sections, and hydraulic corrections to account for convergingdiverging pore cross-sections along the pore length. This method has been applied to a range of sandstone and carbonate rocks having permeabilities that varied over three orders of magnitude from 1-1000 $\mathrm{mD}\left(10^{-15}-10^{-12}\right.$ $\left.\mathrm{m}^{2}\right)[7,10,11]$ and it was found that the predictions were generally accurate to within a factor of two. The objective of this study is to investigate, for the first time, the feasibility of this method to predict the permeabilities of 
a range of cement-based materials which generally have a wider range of pore size, a more complex pore structure and lower permeabilities than rocks.

\section{Modelling}

\subsection{Conductance of individual pores}

The pore structure will be idealised as a network of tubes arranged in a simple cubic lattice. To determine the permeability of this network, we need to know the volumetric flow rate through each pore, which we will estimate using hydraulic radius approximation. Consider a single pore tube of arbitrary cross-section with a pressure gradient $\Delta P / L$ along its length. The volumetric flow rate through this pore can be written as $Q=C \Delta P$ / $\mu L$ according to Poiseuille's law, where $\mu$ is the fluid viscosity and $C$, which has dimensions of $L^{4}$, is referred to as the pore hydraulic conductance. For a cubic lattice network of identical pores, each having a conductance $C$, the permeability of the network would be $k=n C$, where $n$ is the number of pores per unit area in a plane perpendicular to the lattice direction [10].

For an idealized case of a cylindrical tube of radius $r$ and constant cross section, the volumetric flow rate is given by the Hagen-Poiseuille's equation as $Q=\pi r^{4} \Delta P / 8 \mu L$. The hydraulic conductance is therefore $C=\pi r^{4} / 8$. Given that the cross-sectional area of the cylindrical tube is $A=\pi r^{2}$ and the perimeter is $\Gamma=2 \pi r$ the hydraulic conductance can also be written as

$C=\frac{\pi r^{4}}{8}=\frac{A R_{h}^{2}}{8}=\frac{A^{3}}{2 \Gamma^{2}}$

where $R_{h}$ is the hydraulic radius, defined by $R_{h}=2 A / \Gamma$. The equation above can also be used to estimate the conductance of an arbitrary shaped pore (i.e., the so-called hydraulic radius approximation [12]) if the area and perimeter of the pore are known or measured (e.g. from images). This approach avoids the use of computationally intensive procedures to solve the actual flow equation for non-cylindrical pores. The error due to this approximation has been calculated for various pore shapes [12, 9], including actual pores of rocks from SEM micrographs by numerical simulations [5, 8]. These studies showed that the approximation does not systematically overpredict or underpredict conductance, and that the error is generally within $\pm 30 \%$ of the exact conductance.

\subsection{Effect of converging-diverging cross-section}

The hydraulic radius approximation is valid for a pore with constant cross-section along its length. However, the cross-section of a real pore is non-uniform, so this effect must be taken into account. We can consider the simple case of a pore with radius that varies as a sinusoidal function of the position along the pore such that $r(z)=\langle r\rangle[1+\varepsilon \sin (2 \pi z / \lambda)]$, where $\varepsilon$ is the amplitude and $\lambda$ is the wavelength of the radius variation. The hydraulic conductance of this idealised pore can be found by integrating the Hagen-Poiseuille equation along the 
tube length to obtain the overall pressure drop, and expressing the result in the general form $Q=C \Delta P$ / $\mu L$, which in general gives

$$
C=\frac{\pi}{8}\left\langle r^{-4}\right\rangle^{-1}
$$

where the brackets $\langle\cdot\rangle$ represent the average value along the length of the pore tube. If the conductance of this pore is to be determined from a single random section through the pore, the best estimate would be found (on average, over a collection of such pores) by multiplying this conductance by a constriction factor $f$, defined as the ratio of conductance of the sinusoidal pore to that of the cylindrical pore with an average radius $\langle r\rangle$. The constriction factor can be expressed as [10, 13]

$$
\frac{1}{f}=\frac{\left\langle r^{-4}\right\rangle}{\langle r\rangle^{-4}}=\frac{1}{\lambda} \int_{0}^{\lambda}[1+\varepsilon \sin (2 \pi z / \lambda)] d z
$$

which in this case leads to

$$
f=\frac{256 \rho^{7 / 2}}{(1+\rho)^{4}\left(5 \rho^{3}+3 \rho^{2}+3 \rho+5\right)}
$$

where $\rho$ is the ratio of the minimum to maximum pore radius, $\left(r_{\min } / r_{\max }\right)$. The above expression is strictly valid for pores with a sinusoidal profile, but similar analyses using sawtooth and step-function profiles show that the constriction factor is relatively insensitive to the details of the radius variation, except for very small values of $r_{\min } / r_{\max }[10]$. We note that it would be difficult to derive a generalised constriction factor for irregular pores, and that this method is only a first order approximation to the more fundamental Navier-Stokes equations.

The constriction factor can be calculated with Eq. 4 using a suitable estimate of $r_{\min } / r_{\max }$, which we will assume to be 0.43 for all samples, giving a constriction factor of 0.44 . We applied the same constriction factor to all samples for the sake of consistency, and since a more refined approach would probably require threedimensional imaging, which we aim to avoid. The estimate for $r_{\min } / r_{\max }$ is based on the average of the value for a pore space formed by a simple cubic arrangement of mono-dispersed spheres $(=0.57)$ and the value for a more compact hexagonal packing $(=0.29)$ obtained by Cargill [14]. Lock et al [10] showed that a $\pm 10 \%$ change in $r_{\text {min }} /$ $r_{\max }$ from the chosen value will produce a change in $f$ of about $20 \%$.

\subsection{Stereological corrections}

The area and perimeter of every pore in the microstructure will be measured from backscattered electron images (see Section 3.3). These pores are assumed to form an interconnected cubic lattice, where the conductance of each pore is calculated using Eq. 1 along with the constriction factor in Eq. 4. However, a two-dimensional image will sample the pore structure at an arbitrary plane. Because the imaging plane intersects the pore axis at a 
random angle that is not likely to be normal to this axis, the pore area and perimeter measured from images will generally be larger than the actual values. This will cause the calculated pore conductances to be overestimated. Thus, it is necessary to apply stereological corrections factors to the raw image data in order to determine the 'actual' pore areas and perimeters.

Lock et al. [10] derived an approximate stereological correction factor that converts the measured values of the hydraulic conductance $C_{\text {meas }}$ to the 'actual' values $C_{a c t}$. A cylindrical pore with radius $r$ and constant crosssection intersected by the image plane at an angle $\theta$ relative to the pore axis will appear as an ellipse on the image. Lock et al. [10] showed that the ratio of the actual to the measured conductance can be expressed as

$\frac{C_{a c t}}{C_{\text {meas }}}=\frac{\cos \theta\left(1+\cos ^{2} \theta\right)}{2}$

A stereological correction factor can then be derived by averaging the over-estimation in the conductance (Eq. 5) for all possible angles assuming that the pores are randomly oriented with respect to the imaging plane. This yields

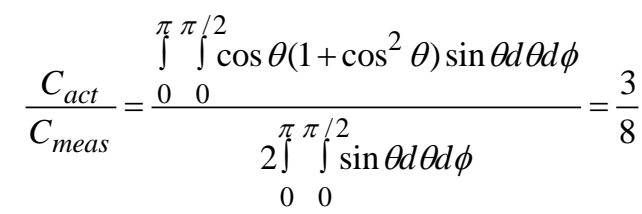

where $\theta$ and $\phi$ are the angles of spherical coordinates.

Lock et al. [10] also considered the overestimation of the number density, i.e. the number of pores per unit area, as a result of making measurements on an arbitrary two-dimensional image that is not likely to be in a plane perpendicular to a lattice direction. To evaluate this effect, the pore structure is again idealised as a hypothetical cubic lattice and the number of pore intersections made by an arbitrary slice $\rho_{\text {slice }}$ is related to the 'actual' number density in a lattice direction $\rho_{a c t}$. The result of averaging over all possible angles yields

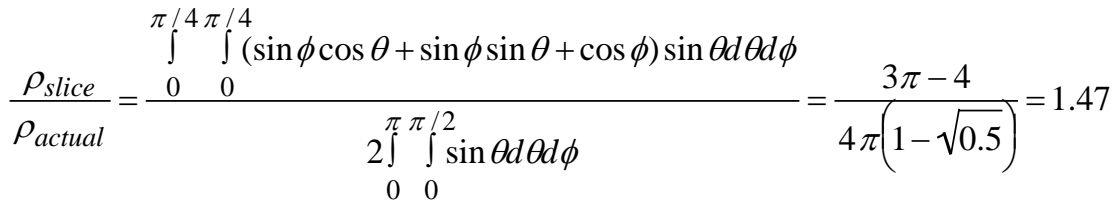

\subsection{Network model and effective medium approximation}

In the final step, the values of all the individual pore conductances are used to compute the macroscopic conductance of the network. Given that the topology of the network is known (i.e., an assumed cubic lattice), the flow equation $Q=C \Delta P / \mu L$ is applied to each pore tube to relate flux to the pressure drop and mass conservation is 
applied at each node to yield a large set of linear equations, which can be solved explicitly, but requires extensive computations. The total flow rate is obtained by summing up the fluxes through every pore that exit the lattice at the outflow face and the permeability is calculated using Darcy's law from the total flow rate and overall pressure drop.

An alternative approach to this exact network calculation is the effective medium approach where each pore conductance $C_{i}$ in the network is replaced by a conductor having an effective value $C_{e f f}$. A widely used method of estimating the effective conductance is Kirkpatrick's approximation [15] by solving the following equation

$\sum_{i=1}^{N} \frac{C_{e f f}-C_{i}}{[(z / 2)-1] C_{e f f}+C_{i}}=0$

where $\mathrm{z}$ is the co-ordination number that represents the number of pores connected at each node and $N$ is the total number of pores. For a cubic lattice, $z=6$. Note that the effective medium approach uses the same input data as the exact network calculation, but requires the solution of only one nonlinear algebraic equation. Eq. 8 is an $N$ thorder polynomial that can be solved using a standard root-finding algorithm such as the bisection or NewtonRaphson procedure. Kirkpatrick’s approximation effectively transforms a heterogeneous medium to a hypothetical homogeneous one with the same topology, but in which all the conductances have a single effective value, thus simplifying the computation of macroscopic properties [16, 17]. We note that Lock et al. [10] evaluated and compared the results from Kirkpatrick's approximation with exact solutions of the network equations, and errors of less than 3\% were observed.

Finally, the permeability of the porous medium $k$ is calculated from the effective pore conductance $C_{e f f}$. This is carried out by considering the total flow $Q$ through a plane that slices the cubic lattice, perpendicular to one of the lattice directions. This plane has an area $A$ and contains $N$ number of pores, which we can estimate from image analysis and the stereological correction factor in Eq. 7. The total flow rate through this plane is given by $Q=N C_{e f f} \Delta P / \mu L$. Comparing this to Darcy's law yields

$k=\frac{N C_{e f f}}{A}$

The above equation can be directly applied to estimate the permeability of neat cement paste samples. Mortar samples contain sand particles, which we will model as non-conducting spherical inclusions randomly embedded in a continuous porous cement paste matrix. The paste matrix is treated as a uniform phase for simplicity, and no special treatment is accorded to the aggregate-paste ITZ, consistent with the findings of Wong et al. [2]. Using the classical Maxwell's approximation, the permeability of a mortar $k_{m}$ is estimated as

$k_{m}=\frac{\left(1-V_{a}\right)}{\left(1+0.5 V_{a}\right)} k$

where $V_{a}$ is the sand volume fraction, and $k$ is the permeability of the cement paste. 


\section{Experimental}

\subsection{Samples}

Our intention is to test the method on a range of cement pastes and mortars with different microstructural characteristics. Seven neat cement pastes and nine mortars were prepared according to the proportions shown in Table 1. The main mix variables were the free water-to-cement mass ratio $(0.25,0.30,0.35,0.40$ and 0.50$)$, cement type, sand volume fraction (0-60 \% vol.) and curing age (3-90 days). The cements were CEM I, CEM II with 8\% silica fume (SF) and CEM III with 60\% slag (GGBS). Mortars were prepared using medium graded Thames Valley sand $(<5 \mathrm{~mm})$. A range of sand content was used to vary the amount of cement paste and aggregate-paste interfaces. Tap water was used as batch water and a sulphonated naphthalene-based superplasticizer was added to improve workability of mixes with low w/c ratio, containing silica fume or high sand content.

Materials were mixed in a pan mixer and cylindrical samples of $100 \mathrm{~mm}$ diameter and $250 \mathrm{~mm}$ length were cast by compaction in three layers using a vibrating table. The samples were covered with plastic sheets and wet hessian for the first 24 hours, demoulded, sealed in cling-film and polythene bags, and left to cure at $20 \mathrm{C}$. After a specified curing period, each cylinder was cut with a diamond saw from the centre to produce three $50 \mathrm{~mm}$ thick disks for oxygen permeability testing and three $8 \mathrm{~mm}$ thick disks for microscopy. The disks were subsequently towelled dry and preconditioned in $\mathrm{CO}_{2}$-free incubators.

The disks were preconditioned by gradual drying at $20 \mathrm{C} 52 \%$ r.h. for one week, then at $35^{\circ} \mathrm{C} 30 \%$ r.h. for a nother week and finally at 50C $10 \%$ r.h. until the mass loss was less than $0.01 \% /$ day. The final step provides a similar vapour density and drying condition as $20 \mathrm{C} 55 \%$ r.h, which is sufficient to minimise the effect of moi sture content on oxygen permeability. All pores relevant to transport are emptied at this level as further drying produced negligible increase in transport properties [18-21]. The entire conditioning regime required about 90 days for completion. To avoid subsequent moisture uptake, the disks were cooled and kept in a vacuumed desiccator at room temperature on the day before testing. Checks by weighing before and after cooling in the desiccators found negligible change in mass. The disks were taken out only when required for transport testing. 


\begin{tabular}{cccccc}
\hline Sample & Cement type & $\begin{array}{c}\text { Free w/c } \\
\text { ratio }\end{array}$ & $\begin{array}{c}\text { Cement content } \\
\left(\mathrm{kg} / \mathrm{m}^{3}\right)\end{array}$ & $\begin{array}{c}\text { Sand content, } \\
\mathrm{kg} / \mathrm{m}^{3}(\% \text { vol.) }\end{array}$ & $\begin{array}{c}\text { Curing age } \\
\text { (days) }\end{array}$ \\
\hline \multirow{2}{*}{ Paste } & CEM I & 0.25 & 1746 & - & $3,7,28,90$ \\
& CEM I & 0.30 & 1606 & - & $3,7,28,90$ \\
& CEM I & 0.35 & 1487 & - & $3,7,28,90$ \\
& CEM I & 0.40 & 1384 & - & $3,7,28,90$ \\
& CEM I & 0.50 & 1216 & - & $3,7,28,90$ \\
\multirow{2}{*}{ Mortar } & CEM II (8\% SF) & 0.30 & 1606 & - & $3,7,28,90$ \\
& CEM III (60\% GGBS) & 0.30 & 1606 & - & $3,7,28,90$ \\
\hline CEM I & 0.30 & 1446 & $260(10)$ & 3,90 \\
& CEM I & 0.30 & 964 & $1040(40)$ & 3.90 \\
CEM I & 0.30 & 642 & $1560(60)$ & 3.90 \\
& CEM I & 0.50 & 1330 & $260(10)$ & 3.90 \\
& CEM I & 0.50 & 887 & $1040(40)$ & 3.90 \\
CEM I & 0.50 & 591 & $1560(60)$ & 3.90 \\
& CEM II (8\% SF) & 0.30 & 1446 & $260(10)$ & 3.90 \\
& CEM II (8\% SF) & 0.30 & 964 & $1040(40)$ & 3.90 \\
CEM II (8\% SF) & 0.30 & 642 & $1560(60)$ & 3.90 \\
\hline
\end{tabular}

229

\subsection{Oxygen permeability}

The test involved applying a pressure gradient across the disk and measuring the outflow rate at steady-state condition. Oxygen was selected because it is relevant to reinforcement corrosion, and it does not react with the sample. Gaseous permeation testing is also potentially less damaging as it requires a lower pressure gradient compared to liquid permeation. The test was carried out on bulk material through a cut face to avoid possible gradient effects in the cast surface.

The disk was fitted into a silicon rubber ring in a permeability cell and sealed by axially loading the rubber ring, which expands laterally to provide an airtight seal. Tests were carried out at three input pressures of $0.5,1.5$ and 2.5 bars above atmospheric pressure. Blank testing on a steel disk of similar dimensions at the highest pressure found no measurable outflow, confirming that the seal is effective. At each pressure, five readings were taken to obtain an average flow, from which the apparent permeability coefficient, $k_{g}\left(\mathrm{~m}^{2}\right)$ was calculated from Darcy's equation for compressible fluid flow:

$Q=\frac{k_{g} A}{2 \eta L} \frac{\left(P_{1}^{2}-P_{2}^{2}\right)}{P_{2}}$ 
where $Q$ is the outflow rate $\left(\mathrm{m}^{3} / \mathrm{s}\right), \eta$ is the oxygen viscosity at $20^{\circ} \mathrm{C}\left(=2.02 \times 10^{-5} \mathrm{~N} \mathrm{~s} / \mathrm{m}^{2}\right)$, and $P_{1}$ and $P_{2}$ are the absolute pressures $\left(\mathrm{N} / \mathrm{m}^{2}\right)$ on the inlet and outlet faces respectively. The apparent permeability decreases with applied pressure due to gas slippage, and this effect was corrected using the Klinkenberg method:

$k_{g}=k_{\text {intrinsic }}\left(1-\frac{\beta}{P_{m}}\right)$

where $P_{m}$ (bar) is the mean pressure $\left(=\left(P_{1}+P_{2}\right) / 2\right)$, and $\beta$ is the Klinkenberg constant. The intrinsic permeability coefficient was obtained from the $y$-intercept of the best-fit line of the plot of apparent permeability versus inverse mean pressure. All plots showed a strong linear correlation with a regression coefficient of the least squares fit always greater than 0.99 . Three replicate disks were tested to obtain an average intrinsic permeability coefficient.

\subsection{Image analysis of pore structure}

The pore areas and perimeters were measured on backscattered electron images captured using a field-emission scanning electron microscope. Imaging was carried out on a $40 \times 20 \times 10 \mathrm{~mm}$ block obtained from the centre of each cylinder and conditioned following the procedures described above. The block was then fully resin impregnated, ground with silicon carbide and diamond polished to a 1/4-micron finish [22]. Thirty backscattered electron images were collected from each polished block at $10 \mathrm{keV}$ beam energy. The brightness and contrast settings were calibrated so that the brightness histogram of the recorded image spans the entire range of available grey scale. The same setting was applied to all images for consistency.

The images were obtained at random locations by moving the stage in a grid and stopping at thirty predefined, equally spaced coordinates. For mortar samples, this sampling procedure ensures that the pastes in the 'interfacial transition zone' (ITZ) and 'bulk' region were represented. Thus, the frequency of an image containing areas in the ITZ and bulk paste depends on aggregate distribution and volume fraction. The images were captured at $500 \times$ magnification. Each image contains $2560 \times 2048$ pixels with a pixel spacing of $0.094 \mu \mathrm{m}$. The field of view of each image was $240 \times 192 \mu \mathrm{m}$.

The pores were segmented according to the 'overflow' method [23], whereby the inflection point of the cumulative brightness histogram is taken as the upper threshold level for pores. For mortars, sand particles were first removed from the original image prior to pore segmentation. The segmented pores consist of capillaries, hollowshells and some microcracks. Air voids and bond cracks at the aggregate-paste interface due to damage from sample preparation were carefully excluded from the analysis. Unreacted cement and hydration products were also segmented and measured. Fig. 1 shows an example of the original BSE image and segmented image. 
The segmented pores may consist of single pixel features and small pixel clusters that are probably disconnected, or are artefacts of the imaging and thresholding process. In the study by Lock et al. [10] these artefacts were removed by eliminating features whose areas are less than $1 \%$ of the largest pore in the image. However, pores in cement-based materials are more heterogeneous and have a wider size range than in sandstones or carbonates. Thus, applying this procedure to cement-based materials would remove too many features and could result in a loss of more than $25 \%$ of the total conductance, which is unacceptable. For this study, the smallest features in the dataset are truncated, but only up to the limit where $99 \%$ of the total conductance is retained. We note that this approach is somewhat arbitrary, and further investigation to identify the most suitable method may be required.

\subsection{Predicting permeability}

The procedure for predicting permeability can be summarised as follows. First, the pore structure is segmented from backscattered electron images and the apparent area and perimeter of each pore are measured. Next, the individual pore conductances are calculated using Eq. 1. The stereological correction factor of Eq. 6 to account for the random orientation of the imaging plane with respect to the pore axis, and the sinusoidal constriction factor of 0.44 from Eq. 4 to account for the variation in pore radius along its length are applied. The resulting set of pore conductances is truncated to remove small artefacts and non-conducting features, and is then entered into

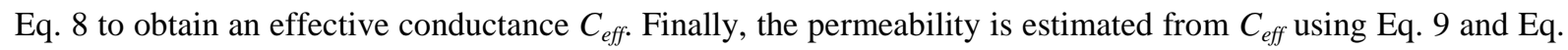
10 (for mortars), after applying the stereological correction factor for apparent number density in Eq. 7.
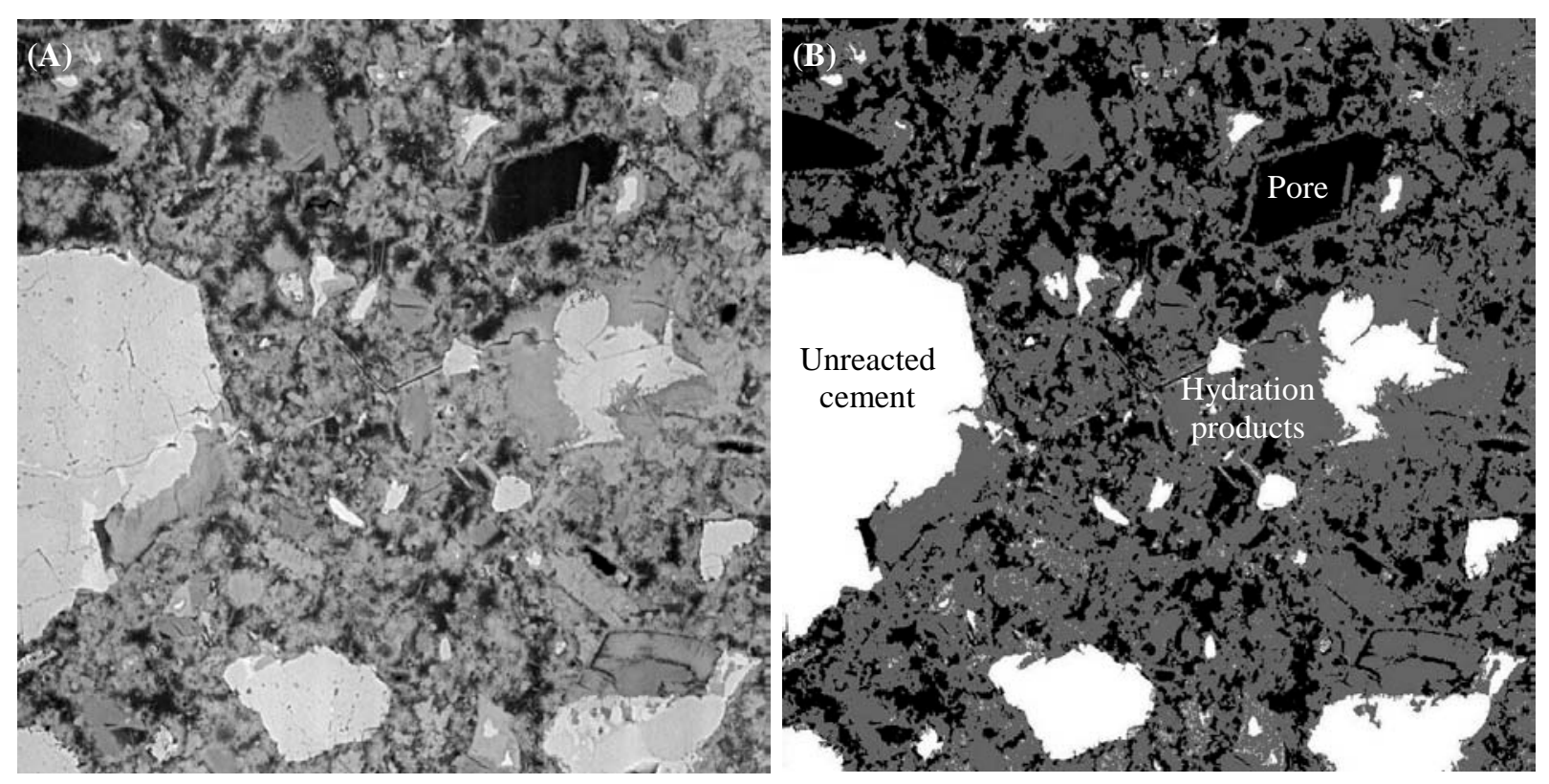

Figure 1 Area-matched backscattered electron micrograph (A) and segmented image (B) of a cement paste highlighting the pores, hydration products and unreacted cement. Field of view is $77 \times 77 \mu \mathrm{m}$. 


\section{Results}

Table 2 summarizes the results from image analysis, permeability measurements and modelling. The measured permeability spans more than two orders of magnitude, from $3 \times 10^{-18} \mathrm{~m}^{2}$ to $5.8 \times 10^{-16} \mathrm{~m}^{2}$. The image measured porosity for all samples range from $5.8 \%$ to $37.5 \%$ and the pore specific surface from $4.5 \mu \mathrm{m}^{-1}$ to $13.0 \mu^{-1}$. As expected, samples with lower w/c ratio and longer curing time had lower porosity and permeability. Samples containing silica fume had the lowest porosity and permeability. For mortars, the measured permeability decreases with increasing sand content. Generally, the specific surface increases as the porosity decreases. The pore size (equivalent spherical diameter) ranges from $0.2 \mu \mathrm{m}$ to $50 \mu \mathrm{m}$. The individual pore conductance spans more than seven orders of magnitude.

Fig. 2 shows the predicted permeability for all samples, compared to the measured values. The dotted lines in Fig. 2 indicate errors of a factor of two in either direction. Most of the data are close to the equality line. From the total of 46 sets of individual data, about $76 \%$ of the predicted values are within a factor of \pm 2 from the measured values, and $98 \%$ are within a factor of \pm 5 . The average percentage error based on absolute values is $63 \%$ and $62 \%$ for paste and mortars, respectively. Generally, the predicted values lie within the upper and lower $95 \%$ confidence limits of the measured values. This suggests that the error in the prediction is no worse than the inherent error in laboratory measurements.

An important issue is the total area that is required to yield representative permeability predictions. Our experience has been that about twenty or more images at a magnification of 500× are required for measuring porosity and unreacted cement content [23, 24]. Fig. 3 shows the change in the predicted permeability with the number of frames analysed for four paste samples at different w/c ratios and curing ages. It can be observed that the predicted permeability settles to a relatively stable value after analysing about thirty images. This suggests that the predictions of the model will not change significantly if more images were to be analysed. Although the total imaged area after thirty frames is only about $1.4 \mathrm{~mm}^{2}$, the total number of individual pores $N$ that is sampled and analysed can be up to $2 \times 10^{5}$, which is probably a sufficiently large dataset to capture a representative size distribution.

(1)
bution. 


\begin{tabular}{|c|c|c|c|c|c|c|c|c|c|}
\hline & $\begin{array}{l}\text { Age } \\
\text { (d) }\end{array}$ & $w / c$ & $\begin{array}{l}\text { Sand } \\
(\%)\end{array}$ & $\begin{array}{l}k_{\text {meas }} \\
\left(\mathrm{m}^{2}\right)\end{array}$ & $\begin{array}{l}\text { Std error } \\
\left(\mathrm{m}^{2}\right)\end{array}$ & $\begin{array}{c}\text { Porosity } \\
(\%)\end{array}$ & $\begin{array}{c}\text { No. of } \\
\text { pores, } \mathbf{N}\end{array}$ & $\begin{array}{l}C_{\text {eff }} \\
\left(m^{4}\right)\end{array}$ & $\begin{array}{l}k_{\text {pred }} \\
\left(\mathbf{m}^{2}\right)\end{array}$ \\
\hline \multirow[t]{20}{*}{ Paste } & \multirow{5}{*}{3} & 0.50 & \multirow{5}{*}{-} & $9.74 \mathrm{E}-17$ & $1.54 \mathrm{E}-17$ & 37.4 & 17473 & $1.69 \mathrm{E}-26$ & $1.45 \mathrm{E}-16$ \\
\hline & & 0.40 & & 8.01E-17 & $9.96 \mathrm{E}-18$ & 28.4 & 36126 & $7.04 \mathrm{E}-27$ & $1.25 \mathrm{E}-16$ \\
\hline & & 0.35 & & $7.58 \mathrm{E}-17$ & 8.10E-18 & 23.3 & 65139 & $3.47 \mathrm{E}-27$ & $1.11 \mathrm{E}-16$ \\
\hline & & 0.30 & & 4.73E-17 & $1.77 \mathrm{E}-18$ & 20.3 & 164739 & $6.57 \mathrm{E}-28$ & 5.32E-17 \\
\hline & & 0.25 & & $1.18 \mathrm{E}-17$ & 9.37E-19 & 14.6 & 120983 & 3.38E-28 & $2.01 \mathrm{E}-17$ \\
\hline & \multirow{5}{*}{7} & 0.50 & \multirow{5}{*}{-} & 7.13E-17 & $1.23 \mathrm{E}-17$ & 30.4 & 38583 & $6.01 \mathrm{E}-27$ & $1.14 \mathrm{E}-16$ \\
\hline & & 0.40 & & 7.22E-17 & $1.37 \mathrm{E}-17$ & 22.8 & 94083 & $1.54 \mathrm{E}-27$ & 7.11E-17 \\
\hline & & 0.35 & & 5.87E-17 & 7.49E-18 & 20.5 & 95214 & $1.43 \mathrm{E}-27$ & $6.70 \mathrm{E}-17$ \\
\hline & & 0.30 & & $4.16 \mathrm{E}-17$ & 3.38E-18 & 19.5 & 104289 & $1.35 \mathrm{E}-27$ & $6.92 \mathrm{E}-17$ \\
\hline & & 0.25 & & $1.17 \mathrm{E}-17$ & 5.84E-19 & 13.8 & 111774 & 7.32E-28 & 4.02E-17 \\
\hline & \multirow{5}{*}{28} & 0.50 & \multirow{5}{*}{-} & $9.57 \mathrm{E}-17$ & $1.58 \mathrm{E}-17$ & 27.5 & 53817 & $3.86 \mathrm{E}-27$ & $1.02 \mathrm{E}-16$ \\
\hline & & 0.40 & & $6.53 \mathrm{E}-17$ & 7.04E-18 & 19.3 & 123336 & 8.33E-28 & 5.05E-17 \\
\hline & & 0.35 & & 5.93E-17 & 4.34E-18 & 18.1 & 97812 & $1.26 \mathrm{E}-27$ & $6.06 \mathrm{E}-17$ \\
\hline & & 0.30 & & 4.67E-17 & $2.71 \mathrm{E}-18$ & 14.9 & 179803 & 4.15E-28 & 3.67E-17 \\
\hline & & 0.25 & & $1.21 \mathrm{E}-17$ & $6.96 \mathrm{E}-19$ & 12.6 & 186545 & 3.64E-28 & 3.34E-17 \\
\hline & \multirow{5}{*}{90} & 0.50 & & $6.82 \mathrm{E}-17$ & $1.47 \mathrm{E}-17$ & 28.6 & 94194 & $1.81 \mathrm{E}-27$ & 8.37E-17 \\
\hline & & 0.40 & & $6.70 \mathrm{E}-17$ & $1.40 \mathrm{E}-17$ & 21.6 & 132542 & 7.69E-28 & $5.01 \mathrm{E}-17$ \\
\hline & & 0.35 & - & 3.91E-17 & 3.02E-18 & 17.0 & 145236 & $6.33 \mathrm{E}-28$ & 4.52E-17 \\
\hline & & 0.30 & & $4.55 \mathrm{E}-17$ & 1.03E-17 & 15.1 & 144969 & $6.30 \mathrm{E}-28$ & 4.49E-17 \\
\hline & & 0.25 & & 8.16E-18 & $1.59 \mathrm{E}-18$ & 13.0 & 235125 & $1.42 \mathrm{E}-28$ & $1.64 \mathrm{E}-17$ \\
\hline \multirow[t]{12}{*}{ Mortar } & \multirow{6}{*}{3} & 0.50 & 10 & 5.75E-16 & $2.98 \mathrm{E}-16$ & 33.7 & 15692 & $4.40 \mathrm{E}-26$ & $2.91 \mathrm{E}-16$ \\
\hline & & 0.50 & 40 & $1.98 \mathrm{E}-16$ & $1.96 \mathrm{E}-17$ & 22.3 & 15237 & $1.74 \mathrm{E}-26$ & $6.51 \mathrm{E}-17$ \\
\hline & & 0.50 & 60 & $6.54 \mathrm{E}-17$ & 3.39E-19 & 15.0 & 10469 & 6.32E-26 & $1.00 \mathrm{E}-16$ \\
\hline & & 0.30 & 10 & $1.18 \mathrm{E}-16$ & 8.80E-18 & 18.3 & 59343 & $4.60 \mathrm{E}-27$ & $1.15 \mathrm{E}-16$ \\
\hline & & 0.30 & 40 & $2.34 \mathrm{E}-17$ & $2.89 \mathrm{E}-18$ & 12.0 & 58198 & 2.33E-27 & 3.33E-17 \\
\hline & & 0.30 & 60 & 8.75E-18 & $1.84 \mathrm{E}-18$ & 8.2 & 47543 & $1.61 \mathrm{E}-27$ & $1.16 \mathrm{E}-17$ \\
\hline & \multirow{6}{*}{90} & 0.50 & 10 & $5.00 \mathrm{E}-16$ & $1.50 \mathrm{E}-16$ & 25.6 & 75713 & $2.01 \mathrm{E}-27$ & $6.40 \mathrm{E}-17$ \\
\hline & & 0.50 & 40 & $1.69 \mathrm{E}-16$ & $1.16 \mathrm{E}-17$ & 14.8 & 48086 & $3.22 \mathrm{E}-27$ & 3.81E-17 \\
\hline & & 0.50 & 60 & 8.31E-17 & $1.30 \mathrm{E}-17$ & 10.8 & 45613 & $3.09 \mathrm{E}-27$ & 2.13E-17 \\
\hline & & 0.30 & 10 & $9.96 \mathrm{E}-17$ & $2.88 \mathrm{E}-18$ & 13.6 & 210354 & $1.09 \mathrm{E}-27$ & $9.70 \mathrm{E}-17$ \\
\hline & & 0.30 & 40 & $2.16 \mathrm{E}-17$ & $2.72 \mathrm{E}-18$ & 9.0 & 199583 & 3.79E-28 & $1.86 \mathrm{E}-17$ \\
\hline & & 0.30 & 60 & $9.62 \mathrm{E}-18$ & $2.49 \mathrm{E}-18$ & 5.8 & 132997 & $2.65 \mathrm{E}-28$ & 5.33E-18 \\
\hline
\end{tabular}




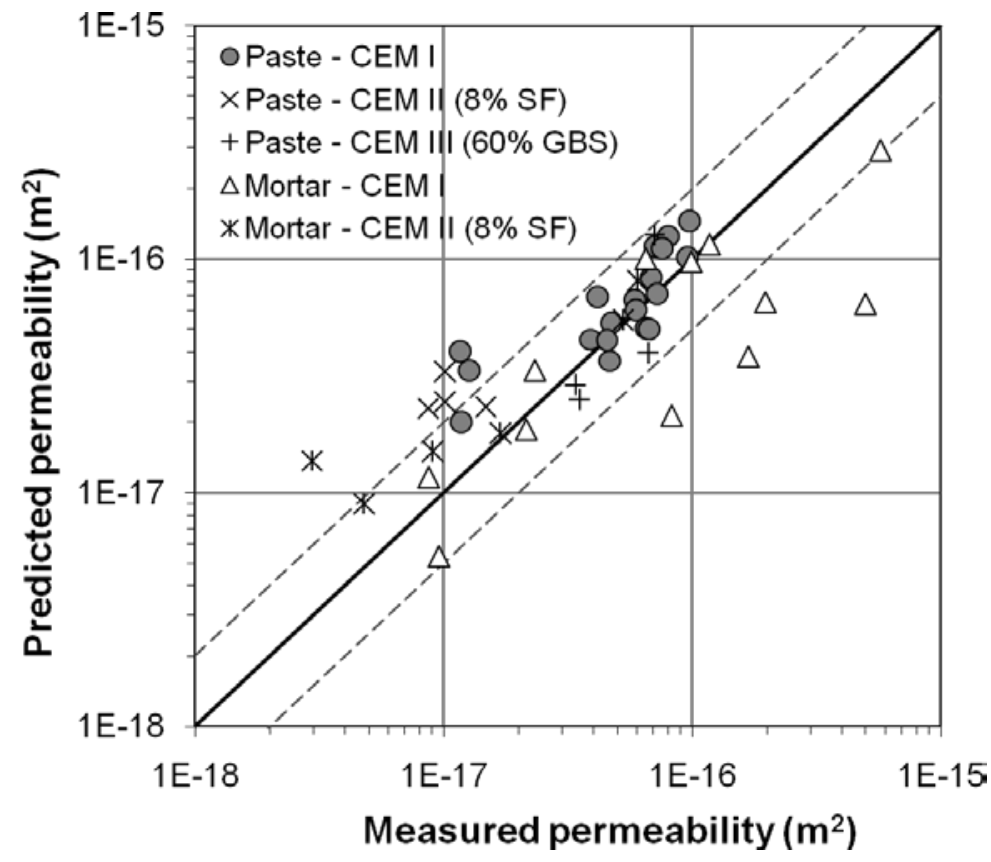

Figure 2 Measured permeability plotted against predicted values for all samples. The dashed lines correspond to errors of a factor of two in both directions.

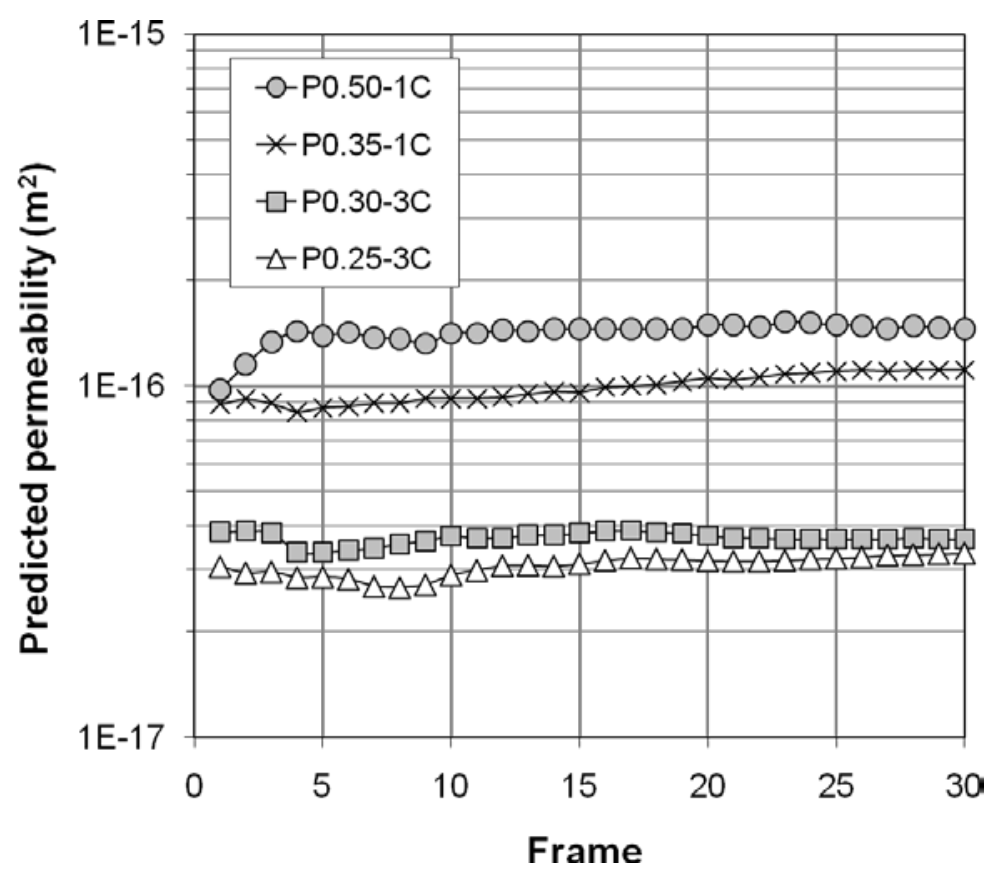

Figure 3 Effect of number of frames analysed on the predicted permeability for selected paste samples. 


\section{Discussion}

The method employed in this study is only slightly less accurate when applied to cement pastes and mortars as compared to sedimentary rocks, despite the fact that the permeabilities of the latter are significantly higher. For example, in the study by Lock et al. [10] on sandstones $\left(k \sim 10^{-14}\right.$ to $\left.10^{-12} \mathrm{~m}^{2}\right), 80 \%$ of the data were estimated to within a factor of \pm 2 with an absolute value error of only $48 \%$. In a subsequent work by Jurgawczynski et al. [11] on sandstones and carbonates $\left(k \sim 10^{-15}-10^{-12} \mathrm{~m}^{2}\right)$, 76\% of the predictions were within a factor of \pm 2 and $90 \%$ were within a factor of \pm 3 .

However, the method is a significant improvement over a previous Kozeny-Carman model that also uses 2D image-derived pore data as inputs [23]. In that study, the permeabilities of mortars were consistently overpredicted by a factor of 5 to 10, with errors increasing with decreasing permeability. A major difference between the two approaches is that the Kozeny-Carman model uses total porosity and specific surface area as inputs, while the current method uses data from individual pores to predict macroscopic permeability via a network model. However, the network model presented by Koplik et al. [8], which is also based on 2D images, consistently over-predicted the permeability of rocks by a factor of ten. The large error from in this model seems to be due to lack of consideration for appropriate stereological correction factors [10].

There is a small tendency for the method to over-estimate slightly in the low permeability range, and underestimate at the high permeability range. This behaviour was also observed by Lock et al. [10] and Jurgawczynski et al. [11]. This could be due to the application of the same co-ordination number for all samples. It is conceivable that samples with high permeabilities are more interconnected and thus have a higher co-ordination number compared to samples with low permeabilities. Indeed, the work by Lindquist et al. [25] using synchrotron X-ray tomography to study the 3D pore structure of sandstones found a positive correlation between permeability and connectivity. Our assumption that $z=6$ for all samples is expected to lead to over-estimation in the low permeability region and under-estimation in the high permeability region. Clearly, more work is needed in this area to characterise and better understand the way in which pores in cement-based materials are connected. Better estimations of the co-ordination number and constriction factors may be obtained by using advanced threedimensional imaging techniques such as FIB-nanotomography, synchrotron computed tomography or confocal microscopy [26-29]. However, it is our intention to use only information derived from 2D images in this work for the sake of simplicity and rapid estimation of permeability.

The model does not accord a special treatment to the aggregate-paste 'interfacial transition zone', but assumes that all the image-segmented pores contribute to transport regardless of their location in the cement paste. This includes the hollow-shell pores, despite the fact that their influence on transport is not well established. On a 2D image, hollow-shell pores appear to be enclosed by a dense layer of hydration product. Using 3D laser scanning confocal microscopy, Head et al. [26] found that the hollow-shell pores are in fact connected to the capillary pore system, but it is still unclear whether the connections are intrinsic, or a result of sample drying. 
It is important to emphasise that the method is based entirely on measured pore data from 2D images as inputs. Although this may at first seem to pose a limitation, we note that Münch and Holzer (2008) recently showed that, if properly interpreted, two-dimensional pore space images can yield essentially equivalent information as is obtained from three-dimensional images. The method contains several adjustable parameters, but the same values for these have been applied to all samples. In this study, we tested the method on cement-based materials with only one modification compared with its application to rocks, which is to the way small artefacts are truncated to account for the difference between the pore structure of cement based materials and rocks. We have used the same stereological corrections, constriction factors, and have again assumed that the pore structure is cubic network with a co-ordination number of six. Based on our analysis, it appears that the method works as well for cement-based materials as it did for sedimentary rocks.

It may be instructive to compare our method to others, such as the Katz-Thompson [30] approach, which is based on percolation theory, and provides rapid permeability estimation using the critical pore size measured from mercury intrusion porosimetry and the formation factor from electrical conductivity measurement as inputs. Several studies have evaluated this method on cementitious materials with varying degrees of success. El-Dieb \& Hooton [31] tested a wide range of cement pastes and concretes, and found errors of several orders of magnitude. They concluded that the Katz-Thompson method is not applicable to cement-based materials. Christensen et al. [32] predicted the permeabilities reported by Nyame \& Illston [33] by measuring the formation factors and critical pore diameters of cement pastes similar to those tested by Nyame \& Illston [33]. The calculated permeabilities were within 1.5 orders of magnitude from measured values, with largest errors for samples with low w/c ratio and long curing ages. In another study, Halamickova et al [34] measured the formation factor, critical pore diameter and permeability from a common set of mortars. Using a constant of 180 (instead of 226 proposed by Katz \& Thompson [30]) they found that the permeabilities of w/c 0.5 mortars can be predicted to within a factor of five, but for the w/c 0.4 mortars, the errors were up to an order of magnitude. This suggests that the method is more applicable for samples with relatively high w/c ratios. However, in another study, Tumidajski \& Lin [35] tested a range of normal concretes and concretes containing fly ash and slag at w/c 0.55 and 0.65 . They found that all calculated permeabilities were two orders of magnitude lower than measured values. More recently, Nokken \& Hooton [36] reviewed the above studies and carried out additional testing. Their findings suggest that the inconsistency in previous studies is due to difficulties and variability in measuring water permeability and formation factor.

Koster et al. [37] simulated the permeation of water through the microstructure of cement paste obtained from 3D microtomographic images. A thinning algorithm was applied to extract the skeleton of the pore space, which was then converted into a network of cylindrical tubes. Laminar flow was assumed in every tube by applying Hagen-Poiseuille's law, and the total flow through the network was then obtained to calculate permeability using Darcy's law. A 'loss coefficient' was included to account for the effects of self-sealing or friction losses on permeability. The authors tested the method on one cement paste (w/c 0.45 , hydration degree 0.67 ) and found excellent agreement with the permeability coefficient measured by Powers [38]. Network models have also been applied to predict permeability of cement pastes from a simulated pore structure such as in the work of Pignat et al. 
[39] and Ye et al. [40]. However, the simulated permeabilities were several orders of magnitude higher than measured values.

Quenard et al [41] applied results from mercury porosimetry and BSE imaging with advanced multi-scale computational modelling techniques involving 3D reconstruction from 2D images and renormalisation using a cubic lattice pore network model to predict air permeability of lime-silica brick, clinker brick and Baumberger sandstone. The permeabilities of the materials investigated ranged from $1 \times 10^{-15} \mathrm{~m}^{2}$ to $3.9 \times 10^{-14} \mathrm{~m}^{2}$, which are slightly higher than the most porous samples tested in the present study. The reconstruction technique and subsequent image-based computations by solving Stokes's equation using a finite-difference method gave computed air permeability to within a factor of 2 of the measured values. Interestingly, the renormalisation technique that represent the pore structure as networks of tubes gave permeabilities that were close to measured values $(<50 \%$ error). Therefore, the accuracy of our approach seems comparable to the most sophisticated and difficult to apply computational models that have been presented.

Finally, it is worth briefly discussing the relationship between our method and the Kozeny-Carman (K-C) model. The K-C model can be derived by assuming a network of pores arranged in a cubic lattice, and using the hydraulic radius assumption to estimate the hydraulic conductivity of each pore, as is done in our model. However, in order to yield the K-C equation, one would also have to make the additional assumption that each pore has the same cross-sectional size and shape. In this case, the "cubic lattice" approximation leads to Eq. 9, and the assumption of identical pores allows one to identify $C_{\text {eff }}$ in Eq. 9 with the conductance of any/each individual pore, as calculated in Eq. 1. Inserting Eq. 1 into Eq. 9 would essentially yield a form of the K-C equation.

However, our model does not assume that each pore has the same geometry or size. On the contrary, we use the area and perimeter of each sampled pore to calculate, from Eq. 1, the hydraulic conductance of that individual pore. But Eq. 9 can then only be used to calculate to overall permeability if we replace this distribution of conductances by a single "effective conductance", for which purpose we use Kirkpatrick's effective medium approximation, Eq. 8. Consequently, the final predictions of our model cannot be written in the form of a K-C equation, mainly because the "averaging" process inherent in the Kirkpatrick equation is highly nonlinear. Moreover, if we were to express the effective conductance that emerges from the Kirkpatrick equation in terms of an "effective pore radius" $r_{\text {eff }}$, the total pore volume represented by a set of pores each having this radius could not be identified with the actual porosity. Hence, although our model can perhaps be thought of as an extension of the K-C model, it cannot be reduced to the K-C model.

We should note that any amount of drying would change the microstructure of cement-based materials, such as by coarsening of the pore structure and inducing micro-cracks from drying shrinkage. However, most concrete structures will experience some form of drying and the associated changes to the microstructure. Therefore, the treatment imposed to our samples, i.e. drying before measuring permeability and pore structure, is not unrealistic given that our aim is to predict transport rates and durability. Testing dried samples gives a conservative estimate 
of permeability for concretes in service. In contrast, measuring permeability of undried and unaltered cement pastes or concretes produces very low permeability values that would seem unrepresentative of the performance of concretes in practice. The samples tested, although covering several w/c ratios, cement type, sand content and age, were by no means exhaustive. More work is required to apply the method to a wider range of samples to substantiate findings. It would also be interesting to study the sensitivity of the predictions to factors such as resolution, image magnification, co-ordination number and the choice of the constriction factor. Extension of the method for the prediction of diffusivity and resistivity, and transport under non-saturated conditions is ongoing.

\section{Conclusions}

A method for estimating permeability, developed by the authors and not previously applied to cement-based materials or materials of such low permeability, is described in this paper. The model is entirely based on pore data obtained from two-dimensional images, requiring only the pore areas and perimeters as input, and involves minimal computational effort. The method has been applied to 46 cement pastes and mortars having a wide range of mix proportions, age and microstructure characteristics. The measured oxygen permeability covered more than two orders of magnitude of data, from $3 \times 10^{-18} \mathrm{~m}^{2}$ to $5.8 \times 10^{-16}$. Results show that about $76 \%$ of the predicted values are within a factor of \pm 2 from the measured values, and $98 \%$ are within a factor of \pm 5 . The average percentage error based on absolute values is $63 \%$ and $62 \%$ for paste and mortars, respectively. The predicted values lie within the 95\% confidence limits of the measured values. These results, which are obtained from a wide range of samples, show that the model can yield predictions with comparable accuracy to complex methods that involve flow simulation through the three-dimensional pore space that are much more computationally intensive and difficult to apply. The simplicity and precision of our approach lends support to the use of images of microstructure as the starting point to predict durability.

\section{Acknowledgements}

We thank Mr. Andrew Morris for his help with the laboratory work. This study was carried out with financial support from the Engineering and Physical Sciences Research Council (EPSRC) through grant EP/F002955/1, which we gratefully acknowledge.

\section{References}

[1] G.W. Scherer, J.J. Valenza, G. Simmons (2007), New methods to measure liquid permeability in porous materials, Cem. Concr. Res., 37 (3) 386-397.

[2] H.S. Wong, M. Zobel, N.R. Buenfeld, R.W. Zimmerman, Influence of the interfacial transition zone and microcracking on the diffusivity, permeability and sorptivity of cement-based materials after drying, Mag. Concr. Res., 61 (8) (2009) 571-589.

[3] J.G. Berryman, S.C. Blair, Use of digital image analysis to estimate fluid permeability of porous materials: Application of two-point correlation functions, J. Appl. Phys., 60 (6) (1986) 1930-1938. 
[4] J.G. Berryman, S.C. Blair, Kozeny-Carman relations and image processing methods for estimating Darcy’s constant, J. Appl. Phys., 62 (96) (1987) 2221-2227

[5] S. Sisavath, X.D. Jing, R.W. Zimmerman, Laminar flow through irregularly-shaped pores in sedimentary rocks, Transp. Porous Media, 45 (2001a) 41-62.

[6] S. Sisavath, X.D. Jing, R.W. Zimmerman, Creeping flow through a pipe of varying radius, Phys. Fluids,

[7] P.A. Lock, X. Jing, R.W. Zimmerman, Comparison of methods for upscalling permeability from the pore scale to the core scale, J. Hydraul. Res., 42 (2004), 3-8.

[8] J. Koplik, C. Lin, M. Vermette, Conductivity and permeability from microgeometry, J. Appl. Phys., 56 (1984) 3127-3131.

[9] E.M. Schlueter, Predicting the transport properties of sedimentary rocks from microstructure, PhD thesis, University of California, Berkeley (1995).

[10] P.A. Lock, X. Jing, R.W. Zimmerman, E.M. Schlueter, Predicting the permeability of sandstone from image analysis of pore structure, J. Appl. Phys., 92 (2002) 6311-6319.

[11] M. Jurgawczynski, P.A. Lock, X.D. Jing, R.W. Zimmerman, Permeability predictions based on twodimensional pore space images, Society of Petroleum Engineers Annual Technical Conference and Exhibition, California (2007) SPE 110472.

[12] F.M. White, Viscous Fluid Flow, McGraw-Hill, New York (1974).

[13] Y. Bernabé, J.F. Olson, The hydraulic conductance of a capillary with a sinusoidal varying crosssection, Geophys. Res. Lett., 27 (2) (2000) 245-248.

[14] G.S. Cargill, Radial distribution functions and microgeometry of dense random packings of hard spheres, In: D.L. Johnson and P.N. Sen (Eds.), Physics and Chemistry of Porous Media, AIP Conference Proceedings, 107, American Institute of Physics, New York (1984) 20-36.

[15] S. Kirkpatrick, Percolation and conduction, Rev. Mod. Phys., 45 (1973) 574-588.

[16] T.C. Choy, Effective medium theory. Principles and applications, Oxford University Press (1999), 182 p.

[17] S. Torquato, Random heterogeneous materials. Microstructure and macroscopic properties, Springer (2001) $701 \mathrm{p}$.

[18] L.J. Parrott (1994), Moisture conditioning and transport properties of concrete test specimens, Mater. Struct., 27, 460-8.

[19] J.P. Ollivier, M. Massat, L. Parrott (1995), Parameters influencing transport characteristics, In: J. Kropp, H,K, Hilsdorf (Eds.), Performance Criteria for Concrete Durability, RILEM Report 12, E \& FN Spon, London, 33-92.

[20] F. Jacobs (1998), Permeability to gas of partially saturated concrete, Mag. Concr. Res., 50 (2) 115-121. 
[21] H.S. Wong, N.R. Buenfeld, J. Hill, A.W. Harris (2007), Mass transport properties of mature wasteform grouts, Adv. Cem. Res., 19 [1] 35-46. doi: 10.1680/adcr.2007.19.1.35

[22] H.S. Wong, N.R. Buenfeld, Patch microstructure in cement-based materials: Fact or artefact? Cem. Concr. Res., 36 (5) (2006c) 990-997.

[23] H.S. Wong, N.R. Buenfeld, M.K. Head, Estimating transport properties of mortars using image analysis on backscattered electron images, Cem. Concr. Res., 36 (2006) 1556-1566.

[24] H.S. Wong, M.K. Head, N.R. Buenfeld, Pore segmentation of cement-based materials from backscattered electron images, Cem. Concr. Res. 36 (2006b) 1083-1090.

[25] W.B. Lindquist, A. Venkatarangan, J. Dunsmuir, T-f Wong, Pore and throat size distributions measured from synchrotron X-ray tomographic images of Fontainebleau sandstones, J. Geophys. Res., 105 (B9) (2000) 21509-21527.

[26] M.K. Head, H.S. Wong, N.R. Buenfeld, Characterisation of 'Hadley’ grains by confocal microscopy, Cem. Concr. Res., 36 (8) (2006) 1483-1489.

[27] E. Gallucci, K. Scrivener, A. Groso, M. Stampanoni, G. Margaritondo (2007), 3D experimental investigation of the microstructure of cement pastes using synchrotron X-ray microtomography ( $\mu \mathrm{CT}), \mathrm{Cem}$. Concr. Res., 37, 360-368.

[28] B. Münch, L. Holzer (2008), Contradicting geometrical concepts in pore size analysis attained in electron microscopy and mercury intrusion, J. Am. Ceram. Soc., 91 [12] 4059-4067.

[29] M.A.B. Promentilla, T. Sugiyama, T. Hitomi, N. Takeda (2009), Quantification of tortuosity in hardened cement pastes using synchrotron-based X-ray computed microtomography, Cem. Concr. Res., 39, 548-557

[30] A.J. Katz, A.H. Thompson (1986), Quantitative prediction of permeability in porous rock, Phys. Rev., B 34 (11) 8179- 8181.

[31] A.S. El-Dieb, R.D. Hooton (1994), Evaluation of the Katz-Thompson model for estimating the water permeability of cement-based materials from mercury intrusion porosimetry data, Cem. Concr. Res., 24 [3] 443-455.

[32] B.J. Christensen, T.O. Mason, H.M. Jennings (1996), Comparison of measured and calculated permeability for cement pastes, Cem. Concr. Res., 31, 277-282.

[33] B.K. Nyame, J.M. Illston (1981), Relationships between permeability and pore structure of hardened cement paste, Mag. Concr. Res., 33 [116] 139-146.

[34] P. Halamickova, R.J. Detwiler, D.P. Bentz, E.J. Garboczi (1995), Water permeability and chloride ion diffusion in Portland cement mortars: Relationship to sand content and critical pore diameter, Cem. Concr. Res., 25 [4], 790-802.

[35] P.J. Tumidajski, B. Lin (1998), On the validity of the Katz-Thompson equation for permeabilities in concrete, Cem. Concr. Res., 28 [5] 643-647. 
[36] M.R. Nokken, R.D. Hooton (2008), Using pore parameters to estimate permeability or conductivity of concrete, Mater Struct, 41, 1-16

[37] M. Koster, J. Hannawald, W. Brameshuber (2006), Simulation of water permeability and water vapour diffusion through hardened cement paste, Comput. Mech., 37 (2) 163-172

[38] T.C. Powers (1958), Structure and physical properties of hardened Portland cement paste, J. Am. Ce-

[39] C. Pignat, P. Navi, K. Scrivener (2005), Simulation of cement paste microstructure hydration, pore

[40] G. Ye, P. Lura, K. van Breugel (2006), Modelling of water permeability in cementitious materials, Mat. Struct. 39, 877-885.

[41] D.A. Quenard, Ke Xu, H.M. Künzel, D.P. Bentz, N.S. Martys (1998), Microstructure and transport properties of porous building materials, Materials and Structures, 31, 317-324. 